\title{
Solubility Determination of Hydroquinone in Dichloromethane, Trichloromethane and Carbon Tetrachloride by Using the Co-solvent Calibration Method
}

\author{
László Kiss ${ }^{1,2}$ · Sándor Kunsági-Máté ${ }^{1,2,3}$
}

Received: 12 February 2019 / Accepted: 16 June 2019 / Published online: 22 October 2019

(c) The Author(s) 2019

\begin{abstract}
In this report, the solubility of hydroquinone (1,4-dihydroxybenzene) was determined in dichloromethane, trichloromethane and carbon tetrachloride at $298 \mathrm{~K}$ by using cyclic voltammetry. By the proposed cosolvent calibration method a mixed solvent was used for the determination of solubility where acetonitrile was the co-solvent. The molar ratios of the two solvents were uniform during the whole procedure both in the calibration solutions and in solutions used for solubility determinations. The solubility of hydroquinone decreased by the decrease of solvent permittivity, which were in dichloromethane $5.683 \mathrm{mmol} \cdot \mathrm{L}^{-1}$, in trichloromethane $2.785 \mathrm{mmol} \cdot \mathrm{L}^{-1}$ and in carbon tetrachloride $0.321 \mathrm{mmol} \cdot \mathrm{L}^{-1}$.
\end{abstract}

Keywords Solubility $\cdot$ Hydroquinone $\cdot$ Cosolvent $\cdot$ Chlorinated hydrocarbons

\section{Introduction}

The knowledge of the solubility of materials in different solvents and solvent mixtures has great significance for many applications [1-5]. Numerous methods can provide a reliable solution for this problem. Gravimetry has had great interest for many years, which is based on the weighing of residual solid material [6]. Particularly, in medical science dimethyl sulfoxide is a solvent which was added to the aqueous solution in low quantity facilitating the dissolution of a large number of compounds [7]. This procedure is complicated as it needs correction equations to calculate the solubility in the aqueous phase containing no dimethyl sulfoxide. Turbidimetry and nephelometry are also used but they are based on the precipitation of material and, due to the possibility of over-saturation, might be inaccurate [8]. The "saturation shake-flask method" is also widespread but it is time consuming [9-17]. The "generator column" method highly facilitates the reaching of solubility

László Kiss

kissl@gamma.ttk.pte.hu

1 Department of General and Physical Chemistry, University of Pécs, Ifjúság Street 6, Pecs 7624, Hungary

2 János Szentágothai Research Center, Ifjúság útja 20, Pecs 7624, Hungary

3 Department of Pharmaceutical Chemistry, University of Pécs, Rókus Street 2, Pecs 7624, Hungary 
equilibrium [18] as the contacting area between the solid material and solvent is very high and the saturation concentration can be measured as usual in chromatography.

Solvent mixtures are used for analytical procedures like in determination of polycyclic aromatic hydrocarbons in oil extracts [19]. There are a large number of works studying the effect of changing the molar ratio of the two and sometimes more solvents on the behavior of a compound [20-22]. There is a solubility determination method that uses a co-solvent introduced into the studied solution [23] but needs a complicated evaluation procedure.

The disadvantage of some methods is the need for a large quantity of solvents and solute, and on the other hand they are sometimes time consuming. The basic problem is usually that there is not a good reference for the exact determination of the unknown saturation concentration. When spectroscopic methods are applied, both the peak position and intensity are affected by the molecular environment of the solute molecules. With the electrochemical methods (mainly voltammetric and amperometric techniques) the composition of the solvent strongly influences the viscosity and consequently the diffusion coefficient that contributes significantly to the signal's magnitude.

In this work, a reliable new method (co-solvent calibration method) is described which is based on ensuring the uniformity of the factors discussed above during the whole procedure.

\section{Experimental}

The chemicals and solvents used were analytical grade (99\%). The supporting electrolyte used in the cyclic voltammetric measurements was tetrabutylammonium perchlorate $\left(\mathrm{TBuClO}_{4}\right)$ with $98 \%$ purity. Water contents of solvents were around $10^{-4} \mathrm{mmol} \cdot \mathrm{L}^{-1}$. The working electrode was a platinum disc (1 mm in diameter) sealed in polyetheretherketone, the counter electrode was a platinum rod and a silver wire served as reference. The electrochemical measurements were carried out with a potentiostat (Dropsens, Spain).

All solutions were prepared in $10 \mathrm{~cm}^{3}$ glass flasks and thermostated at $298 \mathrm{~K}$. The saturated solutions were prepared by adding hydroquinone in excess to the selected solvents. They were thoroughly mixed and ultrasonicated for approximately $5 \mathrm{~min}$ to facilitate the dissolution. Then, the solutions were allowed to sediment but the solid particles did not settle down completely. Due to the presence of solid particles, over-saturation can be avoided. The solid particles had to be separated from the saturated solutions so they were filtrated through a funnel (Sartorius 3hw, average pore size 8-12 $\mu \mathrm{m}$ ). Before all measurements the funnel was thoroughly washed, firstly with distilled water and then with acetone to remove the contaminants and the traces of hydroquinone remained from the previous filtration. After complete evaporation of acetone a new filtration paper was placed into the funnel and the saturated solutions obtained after the filtration were used for the solubility determination.

Another important step was the washing of the solid hydroquinone to separate the microparticles which can go through the pores of filtration paper. The solid material, which was used in the experiments, consisted mainly of macrosized particles. This sample was thoroughly washed with carbon tetrachloride in the filtration paper so microparticles were removed which otherwise could cause error in the determination by penetrating its pores. This fraction was used therefore throughout the work.

When determination of the solubility of a selected material is important, the use of a co-solvent by the calibration method offers many advantages. The basic requirement for 
the co-solvent is that it has to be able to dissolve the solute in high concentration and be miscible with the solvent which is in focus of our interest (primary solvent). In electrochemistry, the permittivity of the co-solvent has to be high enough to dissolve the supporting electrolyte which is necessary to avoid the significant ohmic distortion. During the whole procedure the solvents from the same container should be used as the baseline or background current according to the chosen method, which otherwise could change in the case of different sources increasing the inaccuracy of determination. As the presence of contaminants can modify the solubility of the solute, the solute and primary solvent should have high purity.

The application of the proposed co-solvent calibration method requires some steps that are essential for the highest accuracy:

1. Prepare a stock solution of the solute with the co-solvent whose concentration are adjusted for the preparation of the calibration solution series.

2. Add the supplementary materials (supporting electrolyte, indicator, metal salt, etc.) to the flasks in the necessary quantity.

3. Pipet the exact volume of the primary solvent into all flasks which are consistent with all calibration solutions.

4. Add the exact volumes of the stock solution of solute prepared with the co-solvent to set accurately the desired concentrations of calibration solutions, mix them thoroughly and fill the flasks to the marks with the pure co-solvent.

5. Pipet the same volume of saturated primary solvent to an additional empty flask containing the necessary supplementary materials in the same quantity as for the calibration solutions.

6. Fill the flask containing the saturated primary solvent also with the pure co-solvent to the mark with thorough mixing.

These steps ensure that the solute is at a well-defined concentration in the flasks as the solution volumes are set exactly. The errors caused by the volume changes due to the mixing of the two solvents can be minimized by adding the co-solvent in small portions with mixing. This procedure is applicable by a series of commonly used methods in analytical chemistry (electrochemical, spectroscopic, chromatographic, etc.).

The solubility $(S)$ of the selected material can be calculated by using the next equation:

$$
S=\frac{V(\text { total })}{V(\text { added })} c(\text { meas })
$$

In this equation, $V$ (total) is the total volume of the solution after addition of the necessary amount of the co-solvent (the flask is filled to the mark), V(added) is the exact volume of the added primary solvent, $c$ (meas) is the concentration determined directly from the calibration curve of the calibration solution series made of binary mixtures. The ratio $V($ total $) / V$ (added) expresses the extent of dilution, in other words how many times the saturated solution is diluted. 


\section{Results and Discussion}

In order to determine the solubility of hydroquinone in the three selected chlorinated hydrocarbon solvents the proposed co-solvent calibration method was tested. Hydroquinone is a good model redox-active compound as it undergoes two electron oxidation producing protonated 1,4-benzoquinone [24]. Therefore, electrochemical techniques are suitable to study this compound, so cyclic voltammetry was selected here, which is based on the recording of current peaks when macroelectrodes are used. Acetonitrile was chosen as the co-solvent as it is miscible with all chlorinated hydrocarbon solvents. It has a wide potential window in the positive range making it attractive for electrooxidation studies. On the other hand, it has a relatively high permittivity $(\varepsilon=36)$ which is an essential factor for the electrochemical measurements. The chlorinated hydrocarbons have low dielectric constants: 8.93 for dichloromethane, 4.8069 for trichloromethane and 2.2379 for carbon tetrachloride [25]. The volumes of solvents were $50-50 \%$ of the total value for all chlorinated hydrocarbons. Therefore, the permittivities of mixtures were around 20, which is highly suitable for electrochemical measurements with macroelectrodes. Reliable voltammetric and amperometric measurements can be performed in liquids with permittivity higher than 5 .

The cyclic voltammetric experiments were carried out in the $50-50 \%$ binary solvent mixtures with a $0.1 \mathrm{~V} \cdot \mathrm{s}^{-1}$ scan rate and the concentration of supporting electrolyte was $0.05 \mathrm{~mol} \cdot \mathrm{L}^{-1}$ in dichloromethane and $0.01 \mathrm{~mol} \cdot \mathrm{L}^{-1}$ in trichloromethane and carbon tetrachloride. The potential window was between 0 and 2 V. Figure 1 displays the obtained voltammograms at $298 \mathrm{~K}$ for all three chlorinated hydrocarbons. Keeping the temperature constant ensured constant viscosity and therefore diffusion coefficient so it contributed also to the reproducible current intensities. As can be seen, the anodic peak of hydroquinone appeared between 1 and $2 \mathrm{~V}$ in the solvent mixture of trichloromethane and acetonitrile but peaks appeared also in this potential region in the other two solvents (not shown).

As nothing is known about the saturation concentration it must be estimated previously to fit the concentration range of calibration solutions to it. One possibility for getting an approximate value for it is the taking a voltammogram in the saturated solution with microelectrode and then in a higher permittivity solvent with known concentration of analyte in the presence of supporting electrolyte. Knowing the viscosities of the two solvents the

Fig. 1 Cyclic voltammograms of hydroquinone in 50-50 v/v \% mixtures of trichloromethane and acetonitrile at $298 \mathrm{~K}$ (scan rate $0.1 \mathrm{~V} \cdot \mathrm{s}^{-1}$, supporting electrolyte $0.01 \mathrm{~mol} \cdot \mathrm{L}^{-1} \mathrm{TBuClO}_{4}$ ). The curves for hydroquinone for the different concentrations are: black: $0.5 \mathrm{mmol} \cdot \mathrm{L}^{-1}$; red: $1 \mathrm{mmol} \cdot \mathrm{L}^{-1}$; blue: $1.5 \mathrm{mmol} \cdot \mathrm{L}^{-1}$; dark cyan: $2 \mathrm{mmol} \cdot \mathrm{L}^{-1}$; pink: $2.5 \mathrm{mmol} \cdot \mathrm{L}^{-1}$

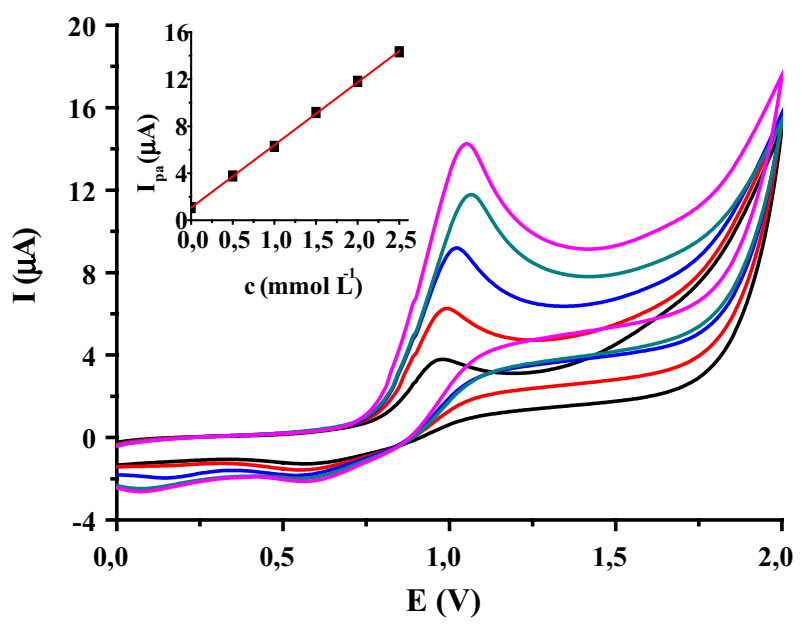


approximate concentration can be estimated. This procedure worked in the case of dichloromethane, therefore the calibration range was between 0 and $10 \mathrm{mmol} \cdot \mathrm{L}^{-1} \mathrm{In}$ the case of trichloromethane well defined voltammogram could not be recorded due to the significant ion association of the supporting electrolyte and in the case of carbon tetrachloride the $\mathrm{TBuClO}_{4}$ did not dissolve at all. Therefore their mixtures prepared with acetonitrile were used and lower concentrations could be estimated. For the two last solvents the calibration range was set between 0 and $2.5 \mathrm{mmol} \cdot \mathrm{L}^{-1}$.

The curves and especially the inset graph show for trichloromethane that the peak currents changed linearly with the concentration of solute. The following linear regressions for the calibration equations were established (for dichloromethane $I_{\mathrm{pa}}=16.593+4.904$ $c\left(\mathrm{mmol} \cdot \mathrm{L}^{-1}\right) \quad\left(\mathrm{R}^{2}=0.999\right), \quad$ for trichloromethane $I_{\mathrm{pa}}=1.072+5.333 \quad c\left(\mathrm{mmol} \cdot \mathrm{L}^{-1}\right)$ $\left(\mathrm{R}^{2}=0.999\right)$, and for carbon tetrachloride $\left.I_{\mathrm{pa}}=0.34+5.583 c\left(\mathrm{mmol} \cdot \mathrm{L}^{-1}\right)\left(\mathrm{R}^{2}=0.998\right)\right)$. The $\mathrm{R}^{2}$ values indicate that the linear fits are excellent. The solubility determinations were carried out for the three solvents through three parallel measurements. The concentrations of hydroquinone were calculated substituting the obtained peak currents into the calibration equations. These values were multiplied by 2 according to the equation mentioned in the "Experimental" section because of the two-fold dilution to get the solubilities. They were averaged for each of the solvents and the average values can be seen in Fig. 2. The saturation concentrations of hydroquinone at $298 \mathrm{~K}$ are $5.683 \pm 0.095 \mathrm{mmol} \cdot \mathrm{L}^{-1}$ in dichloromethane, $2.785 \pm 0.061 \mathrm{mmol} \cdot \mathrm{L}^{-1}$ in trichloromethane and $0.321 \pm 0.006 \mathrm{mmol} \cdot \mathrm{L}^{-1}$ in carbon tetrachloride. The low standard deviations show that the reproducibilities of the determinations are acceptable. According to the expectations, the solubility decreased in parallel with the decreasing dielectric constant (solvent polarity). However, even if the solvent permittivity plays a predominant role in solubility, the solvation can be different for the three chlorinated derivatives of methane. Dichloro- and trichloromethane have hydrogen atoms bound to carbon, being capable of building very weak hydrogen bonds with the solute molecules and thus facilitating their solvation. This type of bond can build up between these hydrogen atoms and nonbonding electron pair of oxygen present in hydroquinone molecules.

Fig. 2 Variation of the solubility of hydroquinone against solvent permittivity

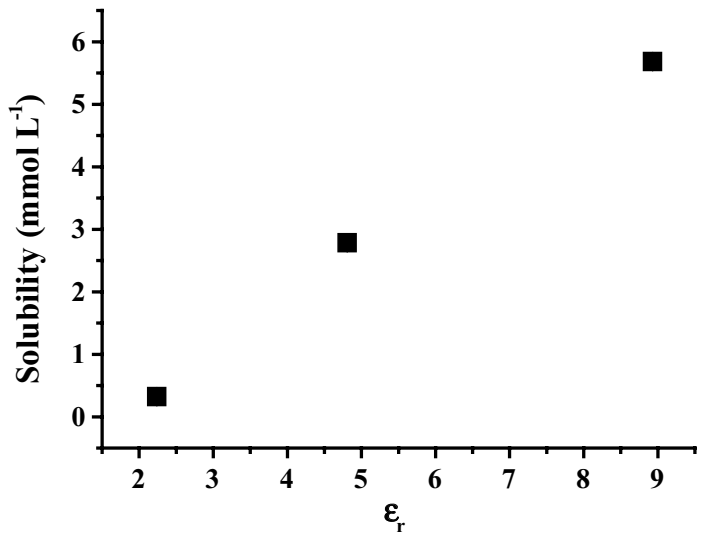




\section{Conclusion}

A new co-solvent calibration method is introduced which is suitable for determination of the concentrations of both solid and liquid materials in a solvent. Furthermore, the method can gain applicability by its procedure, which uses small solution volumes. However, the dilution of samples can be disadvantageous because of the detection limit of selected method, reliable solubility values can be determined. Our plan for the future is the determination of solubilities of selected solid and liquid materials in aqueous and nonaqueous solvents by using the procedure introduced here. This is a promising method, also when the $\mathrm{pH}$ profile of solubility is in focus. It might be applicable by micro-plate techniques by knowing the correction factor for the volume change due to the mixing of two (or more) solvents. It can be used to determine the solubility not only in a solvent but also in solvent mixtures by using ternary or quaternary solvent mixtures for the determination.

Acknowledgements Open access funding provided by University of Pé,cs (PTE). Financial support of the GINOP 2.3.2-15-2016-00022 Grant are highly appreciated.

Open Access This article is distributed under the terms of the Creative Commons Attribution 4.0 International License (http://creativecommons.org/licenses/by/4.0/), which permits unrestricted use, distribution, and reproduction in any medium, provided you give appropriate credit to the original author(s) and the source, provide a link to the Creative Commons license, and indicate if changes were made.

\section{References}

1. Qiushuo, Y., Xiaoxun, M., Long, X.: Solubility in binary solvent mixtures: pyrene dissolved in alcohol + toluene mixtures. Thermochim. Acta 551, 78-83 (2013)

2. Kulkarni, P.P., Jafvert, C.T.: Solubility of $\mathrm{C}_{60}$ in solvent mixtures. Environ. Sci. Technol. 42, 845-851 (2008)

3. Gordon, L.J., Scott, R.L.: Enhanced solubility in solvent mixtures. I. The system phenanthrenecyclohexane-methylene iodide. J. Am. Chem. Soc. 74, 4138-4140 (1952)

4. Escarela, J.B., Bustamante, P., Martin, A.: Predicting the solubility of drugs in solvent mixtures: multiple solubility maxima and the chameleonic effect. J. Pharm. Pharmacol. 46, 172-176 (1994)

5. Patel, S.V., Patel, S.: Prediction of the solubility in lipidic solvent mixture: investigation of the modeling approach and thermodynamic analysis of solubility. Eur. J. Pharm. Sci. 77, 161-169 (2015)

6. Higuchi, T., Connors, A.K.: Phase-solubility techniques. Adv. Anal. Chem. Instrum. 4, 117-212 (1965)

7. Nunez, F.A.A., Yalkowsky, S.H.: Solubilization of diazepam. J. Pharm. Sci. Technol. 52, 33-36 (1998)

8. Avdeef, A., Voloboy, D., Foreman, A.: Dissolution and solubility: pH, buffer, salt, dual-solid, and aggregation effects. In: Testa, B., van de Waterbeemd, H. (eds.) Comprehensive Medicinal Chemisry II. ADME-TOX Approaches, vol. 5, pp. 399-423. Elsevier, Oxford (2007)

9. Zhu, Y.Q., Chen, J., Zheng, M., Chen, G.Q., Farajtabar, A., Zhao, H.K.: Equilibrium solubility and preferential solvation of 1,1'-sulfonylbis(4-aminobenzene) in binary aqueous solutions of $n$-propanol, isopropanol and 1,4-dioxane. J. Chem. Thermodyn. 122, 102-112 (2018)

10. He, Q., Zheng, M., Farajtabar, A., Zhao, H.K.: Solubility and dissolution thermodynamics of cefmetazole acid in four neat solvents and preferential solvation in co-solvent mixtures of (methanol, ethanol or isopropanol) plus water. J. Solution Chem. 47, 838-854 (2018)

11. Blokhina, S.V., Ol'khovich, M.V., Sharapova, A.V., Volkova, T.V., Proshin, A.N., Perlovich, G.L.: Solubility and distribution of bicycle derivatives of 1,3 -selenazine in pharmaceutically relevant media by saturation shake-flask method. J. Chem. Thermodyn. 115, 282-292 (2017)

12. Ishak, H., Stephan, J., Karam, R., Goutaudier, C., Mokbel, I., Saliba, C., Saab, J.: Aqueous solubility, vapor pressure and octanol-water partition coefficient of two phthalate isomers dibutyl phthalate and di-isobutyl phthalate contaminants of recycled food packages. Fluid Phase Equilibr. 427, 362-370 (2016) 
13. Bharate, S.S., Vishwakarma, R.A.: Thermodynamic equilibrium solubility measurements in simulated fluids by 96-well plate method in early drug discovery. Bioorg. Med. Chem. Lett. 25, 1561-1567 (2015)

14. Pobudkowska, A., Domanska, U.: Study of pH-dependent drugs solubility in water. Chem. Ind. Chem. Eng. Q. 20, 115-126 (2014)

15. Domanska, U., Pobudkowska, A., Pelczarska, A., Zukowski, L.: Modelling, solubility and $\mathrm{p} K(\mathrm{a})$ of five sparingly soluble drugs. Int. J. Pharmaceut. 403, 115-122 (2011)

16. Baka, E., Comer, J.E.A., Takacs-Novak, K.: Study of equilibrium solubility measurement by saturation shake-flask method using hydrochlorothiazide as model compound. J. Pharmaceut. Biomed. 46, 335341 (2008)

17. Avdeef, A., Berger, C.M., Brownell, C.: pH-metric solubility. 2: correlation between the acid-base titration and the saturation shake-flask solubility-pH methods. Pharm. Res. 17, 85-89 (2000)

18. Wasik, S.P., Tewari, Y.B., Miller, M.M., Martire, D.E.: Octanol/water partition coefficients and aqueous solubilities of organic compounds. J. Pharm. Sci. 62, 1680-1685 (1981)

19. German, N., Armalis, S.: Voltammetric determination of naphthalene, fluorene and anthracene using mixed water-organic solvent media. Chemija 23, 86-90 (2012)

20. Silva, P.L., Bastos, E.L., El Seoud, O.A.: Solvation in binary mixtures of water and polar aprotic solvents: theoretical calculations of the concentrations of solvent - water hydrogen-bonded species and application to thermosolvatochromism of polarity probes. J. Phys. Chem. B 111, 6173-6180 (2007)

21. Kunsági-Máté, S., Iwata, K.: Electron density dependent composition of the solvation shell of phenol derivatives in binary solutions of water and ethanol. J. Solution Chem. 42, 165-171 (2013)

22. Li, Y., Csók, Z., Kollár, L., Iwata, K., Szász, E., Kunsági-Máté, S.: The role of the solvation shell decomposition of alkali metal ions in their selective complexation by resorcinarene and its cavitand. Supramol. Chem. 24, 374-378 (2012)

23. Avdeef, A.: High-throughput measurements of solubility profiles. In: Testa, B., van de Waterbeem, D.H., Folkers, G., Guy, R. (eds.) Pharmacokinetic Optimization in Drug Research, pp. 305-326. Verlag Helvetica Chimica Acta, Zürich and Wiley-VCH, Weinheim (2001)

24. Eggins, B.R., Chambers, J.Q.: Proton effects in the electrochemistry of the quinone hydroquinone system in aprotic solvents. J. Electrochem. Soc. 117, 186-192 (1970)

25. Lide, D.R.: CRC Handbook of Chemistry and Physics, Chap. 6, 76th edn, p. 173. CRC Press, Boca Raton (1995)

Publisher's Note Springer Nature remains neutral with regard to jurisdictional claims in published maps and institutional affiliations. 